\title{
Beyond Chopsticks: Symmetric Equilibria in Majority Auction Games*
}

\author{
Balázs Szentes and Robert W. Rosenthal ${ }^{\dagger}$
}

September 2001

\begin{abstract}
Majority auction games are simultaneous sealed-bid auctions of identical objects among identical bidders who each want to win a specified fraction ( $>$ half) of the objects. Each bidder receives no benefit from winning less than the specified fraction and no additional benefit from winning more than it. Symmetric equilibria having simple, intuitive forms are shown to exist in first-price, second-price and all-pay versions of such games when the number of bidders is sufficiently large. This contrasts with earlier results for the two-bidder "pure chopstick" majority auction games where the only known equilibria are more complicated.
\end{abstract}

\section{Introduction}

Offshore oil leases and licences for use of parts of the radiofrequency spectrum are two important examples of objects that have been frequently sold through simultaneous auctions. A bidder in simultaneous auctions typically faces an "exposure problem": If her willingnesses to pay for each auctioned object can depend on which other objects she wins, then: by bidding aggressively in the hope of obtaining a collection of objects she most prefers, she runs the risk of overpaying in the event that she wins a less desired set; and by bidding passively she lessens her chances of winning the more desired sets for which she would have been willing to pay more.

${ }^{*}$ We are grateful for research support from the National Science Foundation and for the hospitality of the Department of Political Economy at the University of Siena.

${ }^{\dagger}$ Department of Economics, Boston University 270 Bay State Road, Boston MA 02215, e-mails: szentes@bu.edu and rosentha@bu.edu, respectively. 
That simultaneous auctions give rise to exposure problems is widely appreciated (e.g., Milgrom, 2000), but little is known about the structure of equilibria in games that model them. ${ }^{1}$ In a recent contribution, Szentes and Rosenthal (2001), hereafter SR, made some headway for a particular small class of such models; we constructed symmetric equilibria for two-bidder "pure chopstick" auction games in which three identical objects are sold simultaneously through sealed-bid first-price and second-price auctions. ${ }^{2}$ The preferences of the bidders are such that winning any two of the three objects generates value, while any one is valueless by itself and three are worth no more than two. Even though the games have complete information and identical valuations, the symmetric equilibria constructed for the pure chopstick auctions (one equilibrium for each auction game) are surprisingly complicated: the players randomize on a two-dimensional surface in the three-dimensional bid-vector space.

The pure chopstick constructions were also generalized in SR to games in which the marginal value of the third object is different from zero, but there was no hint there of how to go beyond either two bidders or three objects. In this paper we explore such an extension, studying simultaneous sealed-bid auctions of $n$ objects with $m+1$ bidders who all want to win a number $k(k>n / 2)$ of the objects and receive no benefit when they win fewer than $k$ objects and no additional benefit from winning more than $k$. We call these majority auction games. The pure chopstick games correspond to the special case where $n=3, k=2$, and $m=1$, with the respective payment rules. The constructions here are very different from those in SR, however; this time surprisingly uncomplicated.

While majority auction games with first-price payment rules can be used to model simultaneous sealed-bid auctions and such games with second-price payment rules might be used to model simultaneous ascending auctions, all-pay majority auctions can perhaps best be thought of as models of certain kinds of electoral competitions. In an electoral college scheme, for instance, such as that governing U.S. presidential elections, candidates spend money campaigning in separate state races for electoral college votes. Each state is allocated a fixed number of electors, and a candidate who wins a majority of all electors wins the election. If all states were allocated the same number of electors, if the candidate who spent the most in each state always won the state's electors, and if winning the election were all that motivated the candidates (besides the cost) our all-pay majority auction model would be a perfect description of the game.

\footnotetext{
${ }^{1}$ See, however, Krishna and Rosenthal (1996) and Rosenthal and Wang (1996).

${ }^{2}$ The construction there can easily be adapted to the all-pay case as well.
} 
We begin our analysis by exhibiting a symmetric equilibrium for all the first-price cases satisfying $k=n-1$ and $m>1$. In each such case, the symmetric equilibrium is generated by a probability measure on the $n$-dimensional bid-vector space that has a one-dimensional support. In contrast with the first-price pure-chopstick construction, these equilibria, or at least their supports, are quite intuitive: Each player selects $k$ of the objects at random and then makes equal bids on all $k$, the common level of these bids being chosen according to a particular calculated probability distribution. The player bids zero for the remaining $n-k$ objects. ${ }^{3}$ When $n / 2<k<n-1$, the situation becomes still more interesting. Here independent mixing in the way just described produces indifference for each bidder over the support of the randomized strategy, a necessary condition for equilibrium; but whether it produces an actual equilibrium depends on the size of $m$. We show that if $m$ is sufficiently large (relative to $n$ and $k$ ) the mixture does generate a symmetric equilibrium, but if $m$ is not sufficiently large it does not (as in the pure-chopstick case where $m=1$ ). And in this region of smaller values of $m$, we do not have an alternative equilibrium construction to offer, except in the pure-chopstick case. In addition, unfortunately, our proof technique does not permit us to solve for the minimal $m$ as an explicit function of $n$ and $k$ (except for the case $k=n-1$ where the minimal $m$ is shown to be 2.)

All of this is shown to carry over to second-price and all-pay auction rules. The only modifications needed are in the forms, but not the supports, of the randomizing distributions.

Notwithstanding the extreme assumptions of complete information and identical majoritytype preferences for all bidders and the restriction to sealed-bid rules, our model is able to capture some of the interesting strategic factors associated with the exposure problem and to shed some light on it by showing how equilibrium considerations change as the intensity of the competition changes. In the equilibria introduced here, one bidder always wins exactly $k$ objects, so the outcome is always efficient, and no bidder ever wins more objects than the specified majority. But always there are other bidders who win fewer than $k$ objects. In the equilibrium construction of SR, by contrast, a bidder could win either one, two, or all three objects. In other words, in the pure chopstick equilibria, bidders spread their bids over all the objects (with probability one) in the hopes of winning a majority.

\footnotetext{
${ }^{3}$ Evidence for the intuitiveness of such equilibria: Most theorists whom we asked to guess the form of a symmetric equilibrium of the first-price pure chopstick game began by suggesting randomizing in exactly this way. Unfortunately, as we will see, there can be no such equilibrium in the pure chopstick model (where $m=1$ ).
} 
Here they target a specific subset of the objects having the specified size, guaranteeing at least that they will not win more objects than necessary.

Our new constructions suggest a surprising new role for the number of bidders in an auction. While the idea is commonplace that the presence of more bidders generally helps the seller through increased competition, for the pure-chopstick models two bidders is already sufficient (at the equilibria in SR) for the seller to extract all the surplus and for the bidders to earn zero expected profits. In those equilibria the bidders randomize over a relatively large part of the bid-vector space. In the newly constructed equilibria, the bidders also earn zero expected profits. But evidently when the number of bidders becomes sufficiently large, the nature of the competition changes to one in which the bidders bid in an intuitive fashion on a minimal set that has value. Bidding in this way when there are not sufficiently many competitors presents an opportunity for a rival to exploit, and that is part of the reason why the more complicated constructions are necessary for the pure chopstick auctions. ${ }^{4}$

We begin in Section 2 with a description of the model and the general one-dimensional support construction for the first-price cases, and with some preliminary observations. Section 3 proves that the construction generates equilibria for the first-price cases where $k=n-1$ and $m>1$. The proof here is relatively straightforward, involving a detailed calculation when $m=2$ and an induction argument for $m>2$. The proof that the first-price construction works for general $k$ whenever $m$ is sufficiently large is the subject of Section 4. This proof is indirect and complicated, and pieces of it are relegated to an appendix. The reason we resort to an indirect proof method when $k<n-1$ is that the combinatorics become overwhelming when attempting to prove directly that there are no profitable deviations from the strategy profile in question. To get around the combinatorics, we work instead with a particular transformation of the bid vectors which when $m$ is large serves to push the problematic deviations to a neighborhood of the transformed support of the strategy, where they can be more easily dealt with. Even with this trick, however, a fair amount of detailed argument is needed. In Section 5 we show that simple modifications of the arguments in Sections 3 and 4 produce the analogous results for the second-price and all-pay cases. Section 6 contains a number of additional

\footnotetext{
${ }^{4}$ These interpretive remarks implicitly assume that there are at least no symmetric equilibria other than those constructed, which we conjecture to be so in all cases where $n / 2<k<n$. That there are asymmetric equilibria in addition is easy to verify, but we do not view these as realistically playable anonymous symmetric environments. See Section 5 for the asymmetric equilibria and more on interpretation.
} 
observations.

\section{Preliminaries for the First-Price Cases}

There are $m+1$ bidders competing in simultaneous sealed-bid first-price auctions for $n$ identical objects listed in some specified order $(m \geq 1, n \geq 3)$. A pure strategy for a bidder is an element of $R^{n}$, interpreted as an ordered list of bids. The highest of the $m+1$ bids for each object wins that object at the cost of the bid. If a bidder wins $q$ objects, his payoff is $u(q)$ (his revenue) less the sum of his winning bids (his cost), where

$$
u(q)= \begin{cases}0 & \text { if } q<k \\ k & \text { if } q \geq k .\end{cases}
$$

$(n / 2<k<n) .{ }^{5}$ For most of the paper the tie-break rule is immaterial; we will specify one as needed. ${ }^{6}$ All bidders desire to maximize their respective expected payoffs.

For $x \in[0,1]$, define

$$
G(x)=\frac{\frac{n-k}{n} x^{\frac{1}{m}}}{1-\frac{k}{n} x^{\frac{1}{m}}} .
$$

Note that $G$ is continuous, strictly increasing, $G(0)=0$, and $G(1)=1$; so $G$ is a cumulative distribution function (cdf). We study through Section 4 the strategy profile under which each bidder independently selects $k$ of the $n$ objects at random and bids $x$ on those $k$ objects and zero on the remaining $n-k$, where $x$ is chosen, again independently across bidders, according to the cdf $G{ }^{7}$ Denote by $F: R^{n} \rightarrow R$ the cdf of the random vector which is generated by one bidder bidding according to this randomization.

Lemma 1 Let $m \geq 1, n \geq 3$, and $n / 2<k<n$. If $m$ bidders randomize independently according to $F$, then the remaining bidder earns zero expected payoff at all pure strategies in the support of $F$.

\footnotetext{
${ }^{5}$ Setting $u$ at $k$ when the quota is satisfied is just a normalization that keeps all bids worthy of attention conveniently in the unit interval.

${ }^{6}$ For an example where the tie-break rule matters, consider the cases where $k=n$. Here there is a pure-strategy equilibrium for every $m$ at which all bidders bid 1 on all objects if the tie-break rule always breaks $n$-way ties by naming some one bidder to be the winner of all the objects. For other tie-break rules, when $k=n$ the existence of equilibria can be problematic (but see Section 5 for a reference to the case $k=n$ and $m=1$, where it turns out that there is a continuum of symmetric equilibria).

${ }^{7}$ In Section 5, where alternative payment rules are studied, the form of the construction is the same except for changes in the function $G$.
} 
Proof: Without loss of generality, take a general pure strategy by the remaining bidder

in the support of $F$ to be $(\underbrace{x, \ldots, x}_{k}, \underbrace{0, \ldots, 0}_{n-k})$, where $x \in[0,1]$. The expected payoff to this strategy is the difference between expected revenue, which is $k$ times the probability of at least $k$ wins, and expected cost, which is $k x$ times the probability that any one bid of $x$ wins. So expected revenue is $k G^{m}(x)$, and expected cost is

$$
k x\left(\frac{n-k}{n}+\frac{k}{n} G(x)\right)^{m} .
$$

The difference between these two expressions is readily seen to be identically zero. ${ }^{8} \|$

The indifference established in Lemma 1 makes the strategy profile a candidate to be an equilibrium. Example 1 shows that the rest of the equilibrium conditions need not be satisfied.

Example 1: The first-price pure chopstick game $(n=3, k=2, m=1)$.

In Example 1 independent randomizations according to $F$ do not constitute an equilibrium. To see this, consider a deviation of the form $\left(1,0^{+}, 0^{+}\right) \cdot{ }^{9}$ Expected revenue from the deviation is two times the probability that one of the $0^{+}$bids matches up against the opponent's zero bid, or 4/3. Expected cost is one. So the expected payoff from the deviation is positive.

In the next two sections we seek sufficient conditions on the parameters for independent randomizations according to $F$ to constitute an equilibrium.

\section{The First-Price Case with $\mathrm{k}=\mathrm{n}-1$}

This section is devoted mostly to a proof of

\footnotetext{
${ }^{8}$ There is an implicit assumption here and elsewhere in the paper that a bid of zero by the deviator always wins when tied with $m$ other zeroes. This turns out to have no effect on the sign of the expected payoff to any bid vector that is used against $m$ bidders randomizing independently according to $F$, and so is irrelevant to the issue of when independent randomizations according to $F$ form an equilibrium. It does serve to make the expected payoff function for such a vector continuous on all of $[0,1]^{n}$, which will serve to simplify some of our later arguments.

${ }^{9} \mathrm{~A}$ bid of $0^{+}$defeats all bids of zero and nothing else. It requires no payment when it wins. Cf. the previous footnote.
} 
Theorem 2 When $k=n-1$ and $m \geq 2, m+1$ independent randomizations according to $F$ constitute an equilibrium for the first-price case.

Proof: In light of Lemma 1, it is sufficient to establish that all deviating bid vectors (i.e., those off the support of $F$ ) generate nonpositive expected payoffs. Any such vector containing bids above one is weakly dominated by a vector in which those bids are replaced by one, and any bid below zero can similarly be replaced by a zero. So we can restrict our attention to deviation vectors from the set $[0,1]^{n}$, and we shall show that these all generate strictly negative payoffs.

Let such a vector be denoted $\mathbf{x}=\left(x_{1}, x_{2}, \ldots, x_{n}\right)$ where, without loss of generality, the coordinates are assumed to satisfy $x_{1} \geq x_{2} \geq \ldots \geq x_{n}$. The probability that any one bid $x_{i}$ wins is

$$
\left(\frac{1}{n}+\frac{n-1}{n} G\left(x_{i}\right)\right)^{m}
$$

The expected cost of the deviation is therefore

$$
\sum_{i=1}^{n} x_{i}\left(\frac{1}{n}+\frac{n-1}{n} G\left(x_{i}\right)\right)^{m},
$$

which after substitution and some manipulation is seen to be $\sum_{i=1}^{n} G^{m}\left(x_{i}\right)$.

To compute expected revenue, let $\mathbf{x}_{-i}$ denote the vector which is $\mathbf{x}$ except that its $i^{t h}$ component $x_{i}$ is replaced by one. Then expected revenue from $\mathbf{x}$ is $(n-1)$ times the probability that the deviation wins at least $(n-1)$ objects, or

$$
(n-1)\left(\sum_{i=1}^{n} F^{m}\left(\mathbf{x}_{-i}\right)-(n-1) F^{m}(\mathbf{x})\right) .
$$

To see this, note that $F^{m}\left(\mathbf{x}_{-i}\right)$ is the probability that all the deviation's bids except possibly the $i^{\text {th }}$ win. Summing these probabilities over $i$ counts $n$ times the event that all $n$ bids win, so all but one of these must be subtracted from the sum.

Next $F(\mathbf{x})$ is the probability that all of the deviation's bids defeat those of a randomly drawn opponent. For this to be the case, either the opponent's random draw from $G$ is below $x_{n}$ (the deviator's smallest bid) or it is between $x_{n-1}$ and $x_{n}$ but the opponent's zero matches up with $x_{n}$. Hence

$$
F(\mathbf{x})=\frac{(n-1) G\left(x_{n}\right)+G\left(x_{n-1}\right)}{n} .
$$


The $F\left(\mathbf{x}_{-i}\right)$ are computed similarly, and, substituting and cancelling, expected revenue becomes

$$
\begin{gathered}
(n-1)\left\{\left(\frac{(n-1) G\left(x_{n}\right)+G\left(x_{n-2}\right)}{n}\right)^{m}+\left(\frac{(n-1) G\left(x_{n-1}\right)+G\left(x_{n-2}\right)}{n}\right)^{m}\right. \\
\left.-\left(\frac{(n-1) G\left(x_{n}\right)+G\left(x_{n-1}\right)}{n}\right)^{m}\right\} .
\end{gathered}
$$

As expected revenue is seen above to depend only on the deviation's lowest three bids and expected cost is increasing in all its bids, we can restrict our search for deviations generating nonnegative expected payoffs to those in which the highest $n-3$ bids are as low as possible; i.e., where $x_{1}=\ldots=x_{n-2}$.

We proceed by induction on $m$. First, for the case $m=2$, expected revenue becomes

$$
\begin{aligned}
\frac{n-1}{n^{2}}\left\{\left((n-1)^{2}-1\right) G^{2}\left(x_{n-1}\right)+\right. & 2 G^{2}\left(x_{n-2}\right)+2(n-1) G\left(x_{n}\right) G\left(x_{n-2}\right) \\
& \left.+2(n-1) G\left(x_{n-1}\right) G\left(x_{n-2}\right)-2(n-1) G\left(x_{n}\right) G\left(x_{n-1}\right)\right\} .
\end{aligned}
$$

Since $\left(G\left(x_{n-2}\right)-G\left(x_{n-1}\right)-G\left(x_{n}\right)\right)^{2} \geq 0$,

$$
\begin{aligned}
\left(G^{2}\left(x_{n-2}\right)+\right. & \left.G^{2}\left(x_{n-1}\right)+G^{2}\left(x_{n}\right)\right) \\
& \geq 2\left(-G\left(x_{n}\right) G\left(x_{n-1}\right)+G\left(x_{n}\right) G\left(x_{n-2}\right)+G\left(x_{n-1}\right) G\left(x_{n-2}\right)\right),
\end{aligned}
$$

with equality only if $G\left(x_{n-2}\right)=G\left(x_{n-1}\right)+G\left(x_{n}\right)$. So expected revenue is no larger than

$$
\begin{aligned}
& \frac{n-1}{n^{2}}\left(\left((n-1)^{2}-1\right) G^{2}\left(x_{n-1}\right)+2 G^{2}\left(x_{n-2}\right)\right. \\
& \left.+(n-1)\left(G^{2}\left(x_{n-2}\right)+G^{2}\left(x_{n-1}\right)+G^{2}\left(x_{n}\right)\right)\right) \\
= & \frac{n-1}{n^{2}}\left((n-1) G^{2}\left(x_{n}\right)+\left((n-1)^{2}+n-2\right) G^{2}\left(x_{n-1}\right)+(n+1) G^{2}\left(x_{n-2}\right)\right),
\end{aligned}
$$

with equality only if $G\left(x_{n-2}\right)=G\left(x_{n-1}\right)+G\left(x_{n}\right)$. But since $x_{n-1} \leq x_{n-2}$, this is in turn no greater than

$$
G^{2}\left(x_{n}\right)+G^{2}\left(x_{n-1}\right)+(n-2) G^{2}\left(x_{n-2}\right),
$$

with equality only if $x_{n}=0$ and $x_{n-1}=x_{n-2}$. And this is in turn no greater than expected cost, with equality only if $x_{n}=0$ and $x_{n-1}=x_{n-2}\left(=x_{n-3} \ldots=x_{1}\right)$. In short, all deviations in $[0,1]^{n}$ generate strictly negative expected payoffs when $m=2$.

Assume now that the theorem is true for $m-1$, and consider the expected payoff $\pi$ from a deviation vector $\mathbf{x}$ for the parameter $m$. We shall make use of the following: 


$$
\begin{aligned}
\frac{\partial \pi}{\partial G\left(x_{n-2}\right)}=(n-1) m & \left\{\left(\frac{(n-1) G\left(x_{n}\right)+G\left(x_{n-2}\right)}{n}\right)^{m-1} \frac{1}{n}\right. \\
+ & \left.\left(\frac{(n-1) G\left(x_{n-1}\right)+G\left(x_{n-2}\right)}{n}\right)^{m-1} \frac{1}{n}-G^{m-1}\left(x_{n-2}\right)\right\} \\
\frac{\partial \pi}{\partial G\left(x_{n-1}\right)}=(n-1) m\left\{\left(\frac{(n-1) G\left(x_{n-1}\right)+G\left(x_{n-2}\right)}{n}\right)^{m-1} \frac{n-1}{n}\right. & \left.-\left(\frac{(n-1) G\left(x_{n}\right)+G\left(x_{n-1}\right)}{n}\right)^{m-1} \frac{1}{n}-G^{m-1}\left(x_{n-1}\right)\right\} \\
\frac{\partial \pi}{\partial G\left(x_{n}\right)}=(n-1) m\left\{\left(\frac{(n-1) G\left(x_{n}\right)+G\left(x_{n-2}\right)}{n}\right)^{m-1} \frac{n-1}{n}\right. & \left.-\left(\frac{(n-1) G\left(x_{n}\right)+G\left(x_{n-1}\right)}{n}\right)^{m-1} \frac{n-1}{n}-G^{m-1}\left(x_{n}\right)\right\} .
\end{aligned}
$$

(Keep in mind that these are only one-sided derivatives along boundaries of the set $\left\{\left(x_{n-2}, x_{n-1}, x_{n}\right): 1 \geq x_{n-2} \geq x_{n-1} \geq x_{n} \geq 0\right\}$.)

By payoff continuity, there is a best deviation from this compact set, with the highest $n-3$ coordinates set equal to $x_{n-2}$. We subdivide the possibilities for best deviation into two cases:

(i) $x_{n-2}=x_{n-1}$. In this case the best accompanying $x_{n}$ is 0 , and the vector $\mathbf{x}$ is in the support of $F$. If $x_{n}>0$, the expected payoff is clearly negative.

(ii) $x_{n-2}>x_{n-1}$. In this case, it must be that $x_{n}>0$; for otherwise it would have to be either the case that $\partial \pi / \partial G\left(x_{n}\right) \leq 0$ at $x_{n}=0$ if $x_{n-1}>0$, which is clearly impossible in the expression for $\partial \pi / \partial G\left(x_{n}\right)$ above when $x_{n-2}>x_{n-1}$, or $\partial \pi / \partial G\left(x_{n-1}\right)+\partial \pi / \partial G\left(x_{n}\right) \leq$ 0 if $x_{n-1}=x_{n}=0$, which is similarly impossible. But if $x_{n}>0$, it must be that $\partial \pi / \partial G\left(x_{n-2}\right) \geq 0$, since otherwise $x_{n-2}$ could be profitably decreased, and it must be that $\partial \pi / \partial G\left(x_{n-1}\right)=\partial \pi / \partial G\left(x_{n}\right)=0$ if $x_{n-1}>x_{n}$ and $\partial \pi / \partial G\left(x_{n-1}\right)+\partial \pi / \partial G\left(x_{n}\right)=0$ if $x_{n-1}=x_{n}$. Either of these implies that the sum of the three partial derivatives must be nonnegative. But adding up the three algebraic expressions for the partial derivatives above yields $m$ times the expected payoff for that same deviation vector $\mathbf{x}$ when the parameter is $m-1$, and this is negative by the induction hypothesis. 
Hence all deviations from $[0,1]^{n}$ generate negative payoffs for all $m \geq 2$.||

Theorem 2 establishes that when $k=n-1$, three bidders are sufficient for the existence of a symmetric equilibrium generated by $F$. For general $k$, however, three bidders are not enough.

Example 2: $n=13, k=7$, and $m=2$.

In Example 2, independent randomizations according to $F$ do not form an equilibrium. To see this, consider a deviation consisting of 6 bids of one and 7 bids of $0^{+}$. The expected cost of this deviation is 6 . The expected revenue is 7 times the probability that at least one of the $0^{+}$bids matches up against two zeroes. But this probability is one minus the probability that none do. The event that none match up against two zeroes can in turn be decomposed into the disjoint events where exactly $j$ of a particular bidder's nonzero bids match against the deviator's $0^{+}$bids for $j=1, \ldots, 7$ and the remaining $(7-j)$ are covered by the second bidder. The probability of this is

$$
\frac{\left(\begin{array}{c}
7 \\
j
\end{array}\right)\left(\begin{array}{c}
6 \\
7-j
\end{array}\right)\left(\begin{array}{c}
13-\left(\begin{array}{c}
7-j \\
j
\end{array}\right. \\
)
\end{array}\right)}{\left(\begin{array}{c}
13 \\
7
\end{array}\right)\left(\begin{array}{c}
13 \\
7
\end{array}\right)}
$$

when $j \in\{1, \ldots, 6\}$, and it is $\left(\begin{array}{c}13 \\ 7\end{array}\right)^{-1}$ when $j=7$. Summing the seven terms yields approximately .1293 , so the expected revenue from the deviation is approximately $7(.8707)>6$, and the deviation is profitable.

The profitable deviations described for Examples 1 and 2 are of a very simple sort. Working with more general deviations is more difficult. That is why we do not know for what $(n, k)$-combinations beyond those covered in Theorem 2 independent randomizations according to $F$ form an equilibrium when $m=2$. As we will see next, however, they all do when the number of bidders is sufficiently large.

\section{The First-Price Case with Large m}

This section is devoted entirely to a proof of

Theorem 3 For fixed $n$ and $k(n / 2<k<n)$, if $m$ is sufficiently large, $m+1$ independent randomizations according to $F$ constitute an equilibrium for the first-price case.

The plan of our proof is to analyze a related game $\Gamma$ among the same $m+1$ players in which the pure strategy space for each player is $[0,1]^{n}$ and the revenue part of each 
player's payoff is again $k$ if at least $k$ of a player's coordinate choices are highest among all players' strategy vectors, and 0 otherwise. The cost part of each player's payoff in $\Gamma$ from the pure-strategy vector $\mathbf{y}=\left(y_{1}, \ldots, y_{n}\right)$ is simply $\sum_{i} y_{i}^{m}$, independently of the others' strategies. Since $G$ is strictly monotone, if we identify each $y_{i}$ with $G\left(x_{i}\right)$ in the original game, the individual player's mixed strategy in question in the original game corresponds in $\Gamma$ to making a uniform- $[0,1]$ draw and placing its realization in $k$ (randomly chosen) out of the $n$ coordinates of the strategy vector with the remaining $n-k$ coordinates set to 0 . Call the cdf of this strategy $E$. Now it is easy to see that $\mathbf{x}=\left(x_{1}, \ldots, x_{n}\right)$ is a profitable deviation from independent randomizations according to $F$ in the original game if and only if $\left(y_{1}, \ldots, y_{n}\right)=\left(G\left(x_{1}\right), \ldots, G\left(x_{n}\right)\right)$ is a profitable deviation from independent randomizations according to $E$ in $\Gamma$ : expected revenues are identical across the games (from monotonicity of $G$ ) and the deviator's expected cost in $\Gamma$ is $\sum_{i} G^{m}\left(x_{i}\right)$, which is the same as the expected cost of $\mathbf{x}$ when all others play the strategy in question in the old game, as in the proofs of Lemma 1 and Theorem $2 .{ }^{10}$

So the plan of our proof is to rule out the possibility of positive payoffs accruing to deviations in $\Gamma$ whenever $m$ is sufficiently large. Of course the support of $E$ in $\Gamma$ is the same as the support of $F$ in the original game and does not depend on $m$, and it is easy to see that the payoff in $\Gamma$ to any vector in this support against $m$ independent selections from $E$ is zero. But, since $G$ depends on $m$, as $m$ changes each $\mathbf{y}$ corresponds to a different $\mathbf{x}$, and this complicates the argument.

There are three main steps in the proof. We will first show (Proposition 6) that because the expected payoff to a deviation in $\Gamma$ happens to be a homogeneous function of degree $m$, if there is a profitable deviation in $\Gamma$ then there is also a profitable deviation in which the highest coordinate is one. We may therefore limit ourselves to the consideration of such deviations. The second step is to show (Proposition 7) that any such deviation vector $\mathbf{y}$ earns a strictly negative expected payoff in $\Gamma$ whenever $m$ is sufficiently large. This is easy to prove (although its analogue would be quite difficult in the original game). It leaves us well short of our goal, however, because it does not provide an $m$ that works uniformly for all such $\mathbf{y}$. The problematic deviations turn out to be those near the top point of the support; accordingly, the (complicated) third step of our argument (Proposition 10) is to show that for some neighborhood of the top point of the support there is an $M$ sufficiently large such that all deviations in the neighborhood are unprofitable whenever $m \geq M$.

\footnotetext{
${ }^{10}$ The alert reader will realize that the proof of Theorem 2 could be expressed equivalently in terms of $\Gamma$ and $E$ as well. It appears not to make the argument there any simpler, however.
} 
Theorem 3 then follows from a compactness argument.

We prepare for the chain of arguments with two lemmas about payoffs in the two games that will prove useful at several places in the development.

Lemma 4 If $\mathbf{x} \in[0,1]^{n}$ satisfies $x_{1} \geq x_{2} \geq \ldots \geq x_{n}$ and $y_{j}=G\left(x_{j}\right)$ for $j=1, \ldots, n$, then

$$
E(\mathbf{y})=F(\mathbf{x})=\frac{\sum_{j=k}^{n}\left(\begin{array}{c}
j-1 \\
j-k
\end{array}\right) G\left(x_{j}\right)}{\left(\begin{array}{c}
n \\
n-k
\end{array}\right)}=\frac{\sum_{j=k}^{n}\left(\begin{array}{c}
j-1 \\
j-k
\end{array}\right) y_{j}}{\left(\begin{array}{c}
n \\
n-k
\end{array}\right)} .
$$

Proof: See Appendix.

Note that since

$$
\sum_{j=k}^{n}\left(\begin{array}{l}
j-1 \\
j-k
\end{array}\right)=\left(\begin{array}{c}
n \\
n-k
\end{array}\right),{ }^{11}
$$

$F(\mathbf{x})$ is a convex combination of the smallest $n-k+1$ coordinates of $\mathbf{y}$. Note also that $G\left(x_{k}\right)$, the largest of these smallest coordinates, has the smallest coefficient, $\left(\begin{array}{c}n \\ n-k\end{array}\right)^{-1}$.

Lemma 5 For $\mathbf{x} \in[0,1]^{n}$, let $S_{j}(\mathbf{x})$ denote the set of subsets of $\left\{x_{1}, \ldots, x_{n}\right\}$ that have cardinality $j, j=1, \ldots, n$. Then the revenue from the deviation $\mathbf{x} \in[0,1]^{n}$ in the original game (resp. $\mathbf{y}=\left(G\left(x_{1}\right), \ldots, G\left(x_{n}\right)\right)$ in $\left.\Gamma\right)$ is

$$
k \sum_{j=k}^{n} c_{j} D_{j}
$$

where each $c_{j}$ is a scalar independent of $\mathbf{x}, c_{k}=1$, and

$$
D_{j}=\sum_{\left\{z_{1}, \ldots, z_{j}\right\} \in S_{j}(\mathbf{x})} F^{m}\left(z_{1}, \ldots z_{j}, 1, \ldots, 1\right)=\sum_{\left\{z_{1}, \ldots, z_{j}\right\} \in S_{j}(\mathbf{y})} E^{m}\left(z_{1}, \ldots z_{j}, 1, \ldots, 1\right) .
$$

Proof: See Appendix.

One immediate consequence of Lemma 5 is that if $2 k-1>n$ (i.e., if a super-majority is needed for a positive revenue), the highest $2 k-1-n$ bids in $\mathbf{x}$ do not affect revenue. Since they do affect cost, we conclude immediately

Corollary The deviation $\mathbf{x}$ (resp. $\mathbf{y}$ ) cannot be payoff maximal in the original game (resp. Г) unless its $2 k-n$ highest bids are equal.

\footnotetext{
${ }^{11} \mathrm{~A}$ proof of this is given implicitly in the proof of Lemma 4.
} 
Lemma 5 will also be used to establish two additional results. First, since (by Lemma 4) $F$ and therefore $F^{m}$ depend only on the $n-k+1$ smallest elements of $\left\{G\left(x_{1}\right), \ldots, G\left(x_{n}\right)\right\}=$ $\left\{y_{1}, \ldots, y_{n}\right\}$ and since $j \geq k \geq n-k+1$, each $D_{j}$ is a polynomial in the coordinates of $\mathbf{y}$ each term of which has degree $m$. Second, if $\mathbf{y}$ consists of $k$ large coordinates and $n-k$ small coordinates (one of the problematic deviations in $\Gamma$ ), then in the revenue expression of Lemma 5 only $D_{k}$ contains a term in which the small coordinates play no role. It is $k E^{m}\left(y_{1}, \ldots, y_{k}, 1, \ldots, 1\right)$, which is a polynomial by Lemma 4 .

We now proceed to the first step of the proof of Theorem 3.

Proposition 6 If there is a profitable deviation in $\Gamma$, then there is also a profitable deviation in $\{1\} \times[0,1]^{n-1}$.

Proof: First, recall that the expected cost of the deviation $\mathbf{y}$ in $\Gamma$ is $\sum_{i} y_{i}^{m}$. Next according to Lemma 5 , the revenue that accrues to $\mathbf{y}$, which is the same as the revenue that accrues to the vector of inverse transforms of the coordinates of $\mathbf{y}$ in the original game, is a linear combination of $m$ th powers of terms that are themselves by Lemma 4 convex combinations of coordinates of $\mathbf{y}$. So both the revenue and cost accruing to the deviation $\mathbf{y}$ are polynomials in the coordinates of $\mathbf{y}$ in which all terms have degree $m$. This implies that the payoff to the deviation $\mathbf{y}$, considered as a function of its $n$ arguments, is homogeneous of degree $m$. Hence rescaling the vector so that its largest coordinate is 1 does not alter the sign of the expected payoff.||

The second step is now easy.

Proposition 7 Suppose $\mathbf{y}=\left(y_{1}, \ldots, y_{n}\right) \in\{1\} \times[0,1]^{n-1}$ and $y_{2} \geq y_{3} \geq \ldots \geq y_{n}$. If $\mathbf{y}$ is not in the support of $F$, then there exists $M$ such that whenever $m \geq M$, the deviation $\mathbf{y}$ has negative payoff in $\Gamma$ when there are $m+1$ players.

Proof: If $y_{1}=y_{2}=\ldots=y_{k}=1$, then revenue is $k$ and the deviation earns negative expected payoff whenever $y_{k+1}>0$. Suppose then that $y_{k}<1$. The probability that it is highest among the selections it matches up against is

$$
\left(\frac{n-k}{n}+\frac{k}{n} y_{k}\right)^{m}
$$

which goes to zero as $m$ gets large. Similarly for $y_{k+1}, \ldots, y_{n}$. Hence expected revenue goes to zero because the probability of at least $k$ wins goes to zero. On the other hand, expected cost is at least one because $y_{1}=1$; so expected payoff is negative for $m$ sufficiently large. $\|$ 
If the proof of Proposition 7 looks suspiciously easy, it is because it is actually a very weak result. Fixing $y_{k}<1$ and letting $m$ get large is equivalent to considering a sequence of bid vectors in the original game that have $n-k+1$ coordinates becoming vanishingly small and hence have vanishingly small probability of producing revenue. On the other hand, since $y_{1}=1$, expected cost is bounded below by one. The proof of Proposition 7 is easy because the deviations it rules out are uninteresting ones. The proof suggests, however, that if a uniform $M$ is to be found the crucial deviations in $\Gamma$ will be those for which $y_{k}$ is near one.

We need two more lemmas to prepare for the third step in the proof of Theorem 3.

Lemma 8 Suppose $1>\lambda_{1} \geq \lambda_{2} \geq \ldots \geq \lambda_{p}>0$ and $\sum_{j=1}^{p} \lambda_{j}=1$. Then

$$
p \sum_{l=1}^{j} \lambda_{l} \geq j \forall j \in\{1, \ldots, p\}
$$

Proof: See Appendix.

Lemma 8 is only needed for the proof of

\section{Lemma 9}

$$
\frac{(n-k+1) \sum_{l=0}^{j-1}\left(\begin{array}{c}
n-1-l \\
n-k-l
\end{array}\right)}{\left(\begin{array}{c}
n \\
n-k
\end{array}\right)} \geq j \forall j \in\{0, \ldots, n-k+1\}
$$

Proof: See Appendix.

We are finally ready for

Proposition 10 There exist $M$ and $\varepsilon>0$ such that if there are more than $M$ bidders,

then all deviations $\mathbf{y}$ in $\Gamma$ with $y_{1}=1$ in the $\varepsilon$-neighborhood of $(\underbrace{1, \ldots, 1}_{k}, \underbrace{0, \ldots, 0}_{n-k})$ generate negative payoffs.

Proof: Consider a deviation y with $1=y_{1} \geq y_{2} \geq \ldots \geq y_{n} \geq 0$, where $y_{k+1}<\bar{\varepsilon} \in(0,1)$ and for some $1 \leq j^{*}<k, y_{k}=y_{k-1}=\ldots=y_{k-j^{*}+1}=w<1$. ( $j^{*}$ is just the number of bids at the level $y_{k}=w$.) For any $\varepsilon>0$, up to permutations of the coordinates, all bids in the $\varepsilon$-neighborhood of the top point of the support have the form of $\mathbf{y}$, where $\bar{\varepsilon}$ is sufficiently small and $w$ is sufficiently close to 1 . We will show that if the number of 
bidders exceeds some uniform bound, the expected payoff from the deviation $\mathbf{y}$ increases strictly with $w$, and hence must be negative (since by continuing to increase bids tied with the $k$ th highest, we come eventually to the situation where the highest $k$ bids are all one, which must produce a nonpositive payoff).

First, from the Corollary we know that expected payoff can be increased unless the top $2 k-n$ bids are equal. But if they are equal and $j^{*} \geq n-k+1$, then the top $k$ bids are already all one and we are finished. Consequently, we restrict attention from now on to the case $1 \leq j^{*}<n-k+1$.

Clearly

$$
\frac{\partial \operatorname{cost}}{\partial w}=j^{*} m w^{m-1}
$$

For revenue, note from Lemma 5 and the discussion following it that

$$
\text { Revenue }=k\left(E^{m}\left(y_{1}, \ldots, y_{k}, 1, \ldots, 1\right)+Q(\mathbf{y})\right),
$$

where the terms that make up $Q$ are all functions of at least one of the small $\mathbf{y}$-coordinates. Now from Lemma 4,

$$
\begin{aligned}
k \frac{\partial}{\partial w}\left(E^{m}\left(y_{1}, \ldots, y_{k}, 1, \ldots, 1\right)\right) & = \\
& m \frac{k \sum_{l=0}^{j^{*}-1}\left(\begin{array}{c}
n-1-l \\
n-k-l
\end{array}\right)}{\left(\begin{array}{c}
n \\
n-k
\end{array}\right)} E^{m-1}\left(y_{1}, \ldots, y_{k}, 1, \ldots, 1\right) .
\end{aligned}
$$

Define

$$
\alpha \equiv \min _{j \in\{1, \ldots, n-k+1\}}\left(\frac{(n-k+1) \sum_{l=0}^{j-1}\left(\begin{array}{c}
n-1-l \\
n-k-l
\end{array}\right)}{\left(\begin{array}{c}
n \\
n-k
\end{array}\right)}-j\right),
$$

which is nonnegative by Lemma 9. It follows that

$$
\frac{k \sum_{l=0}^{j^{*}-1}\left(\begin{array}{c}
n-1-l \\
n-k-l
\end{array}\right)}{\left(\begin{array}{c}
n \\
n-k
\end{array}\right)} \geq j^{*}+\alpha \quad \text { for } j^{*}<n-k+1,
$$

since $k \geq n-k+1$. Furthermore $E\left(y_{1}, \ldots, y_{k}, 1, \ldots, 1\right) \geq w$ since it is an average of numbers with that property; so

$$
k \frac{\partial}{\partial w}\left(E^{m}\left(y_{1}, \ldots, y_{k}, 1, \ldots, 1\right)\right) \geq\left(j^{*}+\alpha\right) m w^{m-1} .
$$


Next define

$$
c \equiv \frac{1}{\left(\begin{array}{c}
n \\
n-k
\end{array}\right)} \bar{\varepsilon}+\left(1-\frac{1}{\left(\begin{array}{c}
n \\
n-k
\end{array}\right)}\right) .
$$

Think of $c$ as a convex combination of $\bar{\varepsilon}$ and the number 1. Observe, again from Lemmas 4 and 5 , that $Q(\mathbf{y})$ is a linear combination of expressions each of which is no larger than $c^{m}$. This is because for $j>k$ at least one small bid brings each $E$ calculation below $c$ and therefore each $E^{m}$ below $c^{m}$. Furthermore, the coefficients in the linear combination depend neither on $\mathbf{y}$ nor on $m$. Therefore $|\partial Q(\mathbf{y}) / \partial w|$ is bounded above by $m c^{m-1}$ times some constant $d$ that is independent of $\mathbf{y}$ and $m$. Combining this with the previous inequality, we get

$$
\frac{\partial \text { revenue }}{\partial w} \geq\left(j^{*}+\alpha\right) m w^{m-1}-d m c^{m-1} .
$$

In light of the formula above for $\partial \operatorname{cost} / \partial w$, it is enough to show that

$$
\alpha m w^{m-1}>d m c^{m-1} .
$$

But if $w \in(c, 1]$, this is true for large enough $m$ and stays true as $w$ increases. Therefore under the hypotheses of Proposition 10 there exists $M$ such that for all $m \geq M$ expected payoffs increase with $w$ until the highest $k$ bids are all one, and this deviation vector in $\Gamma$ has nonpositive expected payoff.||

To conclude the proof of Theorem 3 , let $A(m)$ be the set of vectors $\mathbf{y} \in\{1\} \times[0,1]^{n-1}$ that earn negative payoffs when $\Gamma$ has at least $m$ players. By continuity of the payoff function, each $A(m)$ is open; and by Proposition $7, \cup_{m=1}^{\infty} A(m)$ is $\{1\} \times[0,1]^{n-1}$ except for the vectors in the support of $F$. But Proposition 10 identifies an open set in $\{1\} \times[0,1]^{n-1}$, call it $B$, which contains that support and all of the elements of which earn nonpositive profits. So $\{B, A(1), A(2), \ldots\}$ is an open covering of the compact set $\{1\} \times[0,1]^{n-1}$. The existence of a finite subcover (from compactness) means that there is a finite bound $M^{\prime}$ such that whenever $m \geq M^{\prime}$ all deviations in $\Gamma$ and hence all deviations in the original game generate nonpositive payoffs whenever the game has at least $m$ players.

\section{The Second-Price and All-Pay Cases}

In the previous section we argued that independent randomizations according to the cdf $F$ constitute an equilibrium in the first-price majority auction game if and only if independent randomizations according to $E$ constitute an equilibrium of $\Gamma$. In this section we will show that certain modifications of $E$ generate symmetric equilibria of the second-price 
and all-pay majority auction games, respectively, exactly when $E$ generates a symmetric equilibrium of $\Gamma$; in particular, according to our earlier results, when $m \geq 2$ and $k=n-1$, and when $m$ is sufficiently large. In both cases, the model is the same as that of Section 2 except for the obvious changes in the payment rule. (In the second-price case, the highest bidder for each object wins it and pays the second highest bid. In the all-pay case, the highest bid wins each object, but all bidders pay their bids.)

\subsection{The Second-Price Case}

In $\Gamma$ the strategy $E$ calls for a uniform draw $Z$ from $[0,1]$ to be attached to $k$ of the $n$ coordinates selected randomly. Suppose instead that in the second-price majority auction game each player acts independently according to $E$ except that the bid $b(Z)$ instead of $Z$ is attached to those $k$ coordinates, where

$$
b(z)=\frac{n}{k}\left(\frac{z}{\frac{n-k}{n}+\frac{k}{n} z}\right)^{m-1} .
$$

Call the cdf associated with this strategy $E^{\prime}$ in the second-price majority auction game.

Since $d b(z) / d z>0$ on $[0,1], b$ is strictly increasing, so the expected revenue accruing to any bid vector $\left(z_{1}, \ldots, z_{n}\right)$ used against $m$ independent randomizations according to $E^{\prime}$ is the same as the expected revenue accruing to $\left(b^{-1}\left(z_{1}\right), \ldots, b^{-1}\left(z_{n}\right)\right)$ against $m$ independent randomization according to $E$ in $\Gamma$. The expected cost of bidding $z_{i}$ in the second-price auction is

$$
\int_{0}^{z_{i}} b(s) d Q(s), \text { where } Q(z)=\left(\frac{n-k}{n}+\frac{k}{n} z\right)^{m}
$$

We need only show that this integral equals $z_{i}^{m}$ to establish that $(m+1)$ independent draws from $E^{\prime}$ constitute an equilibrium of the second-price majority auction game whenever $(m+1)$ independent draws from $E$ constitute an equilibrium of $\Gamma$, since $\left(b^{-1}\left(z_{1}\right), \ldots, b^{-1}\left(z_{n}\right)\right)$ is then a profitable deviation in $\Gamma$ against $E$ whenever $\left(z_{1}, \ldots, z_{n}\right)$ is a profitable deviation against $E^{\prime}$. But

$$
\frac{d Q(z)}{d z}=\frac{m k}{n}\left(\frac{n-k}{n}+\frac{k}{n} z\right)^{m-1}
$$

so

$$
\frac{d z_{i}^{m}}{d Q\left(z_{i}\right)}=\frac{d z_{i}^{m}}{d z_{i}} \frac{d z_{i}}{d Q\left(z_{i}\right)}=\frac{n}{k}\left(\frac{z_{i}}{\frac{n-k}{n}+\frac{k}{n} z_{i}}\right)^{m-1}
$$

and so by the Fundamental Theorem of Calculus

$$
\int_{0}^{z_{i}} b(s) d Q(s)=z_{i}^{m} .
$$




\subsection{The All-Pay Case}

The modification of $E$ that works for the all-pay majority auction game is to randomize by making a uniform draw $Z$ as in $E$ but to bid $Z^{m}$ on the $k$ randomly selected objects. As above, the revenue accruing to the vector $\left(z_{1}, \ldots, z_{n}\right)$ against $m$ such randomizations drawn independently in the all-pay game is the same as that accruing to $\left(\left(z_{1}\right)^{\frac{1}{m}}, \ldots,\left(z_{n}\right)^{\frac{1}{m}}\right)$ against $m$ independent draws from $E$ in $\Gamma$. But the expected cost of $\left(\left(z_{1}\right)^{\frac{1}{m}}, \ldots,\left(z_{n}\right)^{\frac{1}{m}}\right)$ in $\Gamma$ is $\sum_{i} z_{i}$, the same as the expected cost of the bid vector $\left(z_{1}, \ldots, z_{n}\right)$ in the all-pay majority auction game.

\section{Additional Remarks}

1. For some parameter combinations we are still in the dark: Except for the case $n=3$, $k=2$, we have no equilibrium constructions when $m=1$, and as $n$ and $k$ get larger we have no equilibrium constructions for small $m$ that are larger than one. Existence of symmetric equilibrium for any of these cases is not in doubt, at least for some tie-break rule (cf. Simon and Zame, 1990), and we suspect that atomless symmetric equilibria always exist for all tie-break rules and for all $m$, but these are likely even more complicated than the one in SR.

2. For the cases $n=3, k=2$, we know that when $m=1$ there area the symmetric equilibria constructed in SR and when $m>1$, there are the symmetric equilibria constructed here. We know of no other symmetric equilibria for these parameter combinations and suspect that there are none. There are asymmetric equilibria for all payment rules however when $m>1$, and at least some of these are easily described. Fix some number $m^{\prime}<m$ of the bidders at the zero vector in $R^{n}$ and have the remaining $m+1-m^{\prime}$ bidders play the symmetric equilibrium of the $m+1-m^{\prime}$ bidder game. Note that all bidders earn zero profits at this asymmetric profile. When $m^{\prime}=m-1$ it was verified in SR that the strategy profile forms an equilibrium. For smaller positive $m^{\prime \prime}$ s, it suffices to check that none of the zero bidders gains by deviating. If one of them deviates to a bid vector on the support of the randomizers, it is easily checked by direct calculation that the payoff is negative, as it is less than the payoff to such a deviation at the equilibrium of the game with $m+1-m^{\prime}$ bidders. If one of them deviates to an off-support vector a similar argument must be made, essentially mimicking the order of the steps in the proof of Theorem 2. For other combinations of $n$ and $k$, we suspect, but have not checked, that asymmetric equilibria 
can be constructed similarly, at least when the reduced-player game is known to have a zero-profit symmetric equilibrium.

3. Except briefly in a footnote, we have so far not mentioned the cases with $k=n$. These cases correspond to games that have a very different structure than the games treated in this paper. With the right tie-break rule, as mentioned, they have pure-strategy Bertrandtype equilibria. More interesting, however, is that even though the exposure problem is avoided if all bidders randomize over equal-bid supports, such randomizations never generate symmetric equilibria. This is easy to see: For the first-price case, for instance, the bottom point of such a support would (unless it were an atom, which would be impossible in equilibrium) generate zero expected payoff with probability one, but intermediate points would generate positive expected payoffs, as they either win all (with positive probability) or none of the objects. And a necessary condition for equilibrium is equal expected payoffs for a player at all points of the support. So it is the existence of an exposure problem when $k<n$, even when all bidders randomize in the pattern of the equilibria of this paper, that renders such structures capable of generating equilibria, which they do when $m$ is sufficiently large.

In related work, Szentes (in preparation) constructs a continuum of atomless, zeropayoff, symmetric equilibria for the cases where $k=n$ and $m=1$ for all tie-break rules. In all these equilibria, bidders randomize on one-dimensional supports, but the paired bids in the support are typically unequal, leading to the possibility of multiple winning bidders all receiving negative payoffs, an event that happens with positive probability.

4. As mentioned in the introduction, all-pay majority auction games can be used as models of the strategic aspects of campaign spending in electoral college contests. Our models correspond to the very special case of equal numbers of votes in each state, each state being a winner-take-all contest, and the winner in each state being the larger spender. Despite all these simplifying assumptions, we have no equilibrium constructions for the two-candidate cases $(m=1)$ except in the simplest case of $k=2, n=3$. Perhaps the difficulty of constructing such an equilibrium even in the simplest setting is related to the high esteem in which successful election strategists are held.

We may simplify our all-pay setting even farther by assuming the candidates have fixed and equal budgets. This turns the game into a "Colonel Blotto" model of the sort originated by Borel (Cf., Borel and Ville, 1938, and Laslier and Picard, 2000). 
5. As in the generalizations of the pure chopstick game in SR, generalizations beyond the stark preference structure of this paper are imaginable and if analyzable would probably go some way toward improved applicability of the model. Indeed, we know of no reallife auction scenarios that are reasonably modeled as pure majority auction games. Even when symmetry and complete information are not far wrong, the payoff forms here seem too extreme. Objects selling at auction rarely have such little value when combined in suboptimal ways that it is reasonable to treat them as literally worthless. And if the assumed payoff forms were correct and known to the seller, she would be inclined to use simpler auction designs anyway. But our purpose has been to expose the structure of symmetric equilibria of a class of games which, while likely inapplicable by themselves, may suggest equilibrium structures in more applicable models. Given the dearth of current knowledge of equilibrium structure of games involving exposure problems, this has seemed to us a fruitful way to proceed. 


\section{Appendix}

Proof of Lemma 4: We need to verify only the middle equality. To do this, decompose the event that all coordinates of $\mathbf{x}$ defeat (including ties) all the bids of a randomly chosen $F$-bidder, $k$ of whose bids are some positive number (say $w$ ) with the remaining $n-k$ being zero, according to the position of that bidder's last $w$ bid.

1. If the last $w$ bid is in position $n$, which can occur in $\left(\begin{array}{l}n-1 \\ n-k\end{array}\right)$ of the $\left(\begin{array}{c}n \\ n-k\end{array}\right)$ equally likely ways for the $n-k$ zero bids to be distributed among the $n$ positions, then all coordinates of $\mathbf{x}$ win whenever $w$ is less than $x_{n}$. The probability of this event is therefore

$$
\frac{\left(\begin{array}{c}
n-1 \\
n-k
\end{array}\right)}{\left(\begin{array}{c}
n \\
n-k
\end{array}\right)} G\left(x_{n}\right) \text {. }
$$

2. If the last $w$ bid is in position $n-1$, which can occur in $\left(\begin{array}{c}n-2 \\ n-k-1\end{array}\right)$ ways (since one of the zeroes is necessarily in position $n$ ), then all coordinates of $\mathbf{x}$ win whenever $w$ is less than $x_{n-1}$. The probability of this event is therefore

$$
\frac{\left(\begin{array}{c}
n-2 \\
n-k-1
\end{array}\right)}{\left(\begin{array}{c}
n \\
n-k
\end{array}\right)} G\left(x_{n-1}\right) \text {. }
$$

$\mathrm{n}-\mathrm{k}+1$. If the last $w$ bid is in position $k$, which can occur in only $\left(\begin{array}{c}n-(n-k+1) \\ 0\end{array}\right)=1$ way, then all coordinates of $\mathbf{x}$ win whenever $w$ is less than $x_{k}$. The probability of this event is

$$
\frac{\left(\begin{array}{c}
k-1 \\
0
\end{array}\right)}{\left(\begin{array}{c}
n \\
n-k
\end{array}\right)} G\left(x_{k}\right) \text {. }
$$

Proof of Lemma 5: First note that in the original game $F^{m}\left(z_{1}, \ldots z_{k}, 1, \ldots, 1\right)$ is the probability that the bids $z_{1}, \ldots, z_{k}$ all win. $D_{k}$ sums such probabilities, and if the events were disjoint, the correct revenue expression would be simply $k D_{k}$. But $D_{k}$ overcounts the probabilities of events in which more than $k$ of the bids from the vector $\mathbf{x}$ win. Correcting for the overcounts involves adding and subtracting scalar multiples of $F^{m}\left(z_{1}, \ldots z_{j}, 1, \ldots, 1\right)$ for $j>k$. The scalars depend only on $j$ and neither on $m$ nor on the specific values of the $\mathrm{x}$-coordinates.

Proof of Lemma 8: Suppose not. Then $\exists j$ such that

$$
p \sum_{l=1}^{j} \lambda_{l}<j \text {, or } \sum_{l=j+1}^{p} \lambda_{l}>\frac{p-j}{p} .
$$


Since there are $p-j$ terms on the left side of the last inequality, at least one, and hence $\lambda_{j+1}$, must exceed $1 / p$. But then the first $j \lambda_{l}$ 's must also, in which case $p \sum_{l=1}^{j} \lambda_{l}>j$, a contradiction.

Proof of Lemma 9: First note that

$$
\sum_{l=0}^{n-k}\left(\begin{array}{l}
n-1-l \\
n-k-l
\end{array}\right)=\left(\begin{array}{c}
n \\
n-k
\end{array}\right)
$$

(as in the proof of Lemma 4) and the successive terms on the left are decreasing in $l$. Lemma 8 therefore implies that

$$
(n-k+1) \frac{\sum_{l=0}^{j-1}\left(\begin{array}{c}
n-1-l \\
n-k-l
\end{array}\right)}{\left(\begin{array}{c}
n \\
n-k
\end{array}\right)} \geq j \quad \forall j \in\{1, \ldots, n-k+1\} .
$$




\section{References}

[1] E. Borel and J. Ville, "Application de la theorie des probabilites aux jeux de hasard," original edition,1938, by Gauthier-Villars, Paris; reprinted in Theorie mathematique du bridge a la portee de tous by E. Borel and A. Cheron, Editions Jacques Gabay, Paris, 1991.

[2] V. Krishna and R. Rosenthal, "Simultaneous Auctions with Synergies," Games and Economic Behavior 17 (1996), 1-31.

[3] J.-F. Laslier, and N. Picard (2000), "Distributive Politics: Does Electoral Competition Promote Inequality?," Discussion paper 2000/22, CORE, Universite Catholique de Louvain.

[4] P. Milgrom, "Putting Auction theory to Work: The Simultaneous Ascending Auction," Journal of Political Economy 108 (2000), 245-272.

[5] R. Rosenthal and R. Wang, "Simultaneous Auctions with Synergies and Common Values," Games and Economic Behavior 17 (1996), 32-55.

[6] L. Simon and W. Zame, "Discontinuous Games and Endogenous Sharing Rules," Econometrica 58 (1990), 861-872.

[7] B. Szentes, PhD thesis, in preparation.

[8] B. Szentes and R. Rosenthal, "Three-Object Two-Bidder Simultaneous Auctions: Chopsticks and Tetrahedra," mimeo, 2001. 